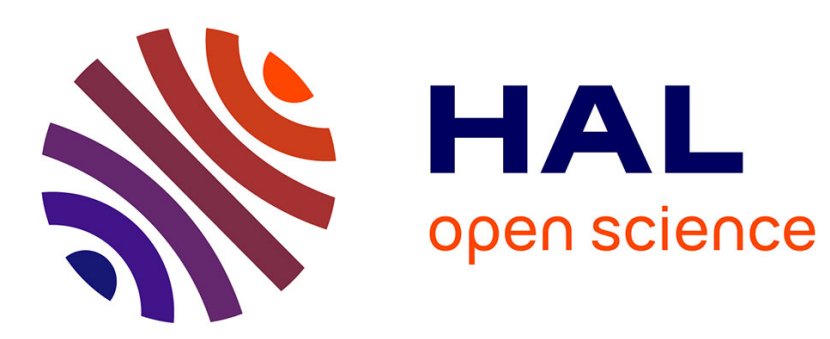

\title{
Quand les voyelles longues et brèves ne tiennent pas en place: la qualité vocalique en allemand L2
}

\author{
Jane Wottawa, Martine Adda-Decker
}

\section{To cite this version:}

Jane Wottawa, Martine Adda-Decker. Quand les voyelles longues et brèves ne tiennent pas en place: la qualité vocalique en allemand L2. XXXIIe Journées d'Études sur la Parole, Jun 2018, Aix-enProvence, France. pp.64-71, 10.21437/JEP.2018-8 . halshs-02130881

\section{HAL Id: halshs-02130881 https://shs.hal.science/halshs-02130881}

Submitted on 16 May 2019

HAL is a multi-disciplinary open access archive for the deposit and dissemination of scientific research documents, whether they are published or not. The documents may come from teaching and research institutions in France or abroad, or from public or private research centers.
L'archive ouverte pluridisciplinaire HAL, est destinée au dépôt et à la diffusion de documents scientifiques de niveau recherche, publiés ou non, émanant des établissements d'enseignement et de recherche français ou étrangers, des laboratoires publics ou privés. 


\title{
Quand les voyelles longues et brèves ne tiennent pas en place : la qualité vocalique en allemand $\mathrm{L} 2$
}

\author{
Jane Wottawa ${ }^{1,2}$ Martine Adda-Decker ${ }^{2}$ \\ (1) LIMSI, CNRS, Université Paris-Saclay, \\ Bât 508, rue John von Neumann, Campus Universitaire, 91405 Orsay, France \\ (2) LPP, UMR 7018 CNRS - U. Paris 3 / Sorbonne Nouvelle, \\ 19 rue des Bernardins, 75005 Paris, France \\ jane.wottawa@univ-paris3.fr, martine.adda-decker@univ-paris3.fr
}

\section{RÉSUMÉ}

En allemand, la distinction entre voyelles longues et brèves n'est pas seulement liée à une différence de durée mais aussi à une différence de qualité dans la plupart des cas. Nous examinons dans quelle mesure les apprenants francophones de l'allemand réalisent une différence de qualité entre les voyelles longues et brèves en comparaison avec des germanophones natifs. Les résultats montrent que de manière générale, les apprenants francophones de l'allemand produisent des voyelles avec un F2 plus élevé que les germanophones natifs. Dans l'espace vocalique, les voyelles peuvent être qualifiées comme étant plus antérieures. Seulement pour la paire [i, I] les apprenants de l'allemand et les germanophones natifs se distinguent sur les valeurs des trois premiers formants. La différence entre [ii] et [I] est bien marquée pour les deux premiers formants chez les germanophones natifs. Les productions des apprenants de l'allemand montrent plutôt des différences sur le F3 pour cette paire vocalique.

\section{ABSTRACT}

\section{When short and long vowels do not keep in place : vowel quality in German L2.}

In German, the distinction between long and short vowels is not only related to a difference in duration but also to a difference in quality most of the time. We investigated to what extend French learners of German realize a quality difference between long and short vowels compared to German native speakers. In general, French learners of German produce vowels with a higher F2 than German native speakers. In the vowel space, vowels can be qualified as more fronted. Only for [i: $\mathrm{I}]$ learners of German and German native speakers differ in the values of the first three formants. The difference between [ii] and [I] is highly marked for the first two formants in German native speakers. The learners' productions rather show differences in $\mathrm{F} 3$ for this vowel pair.

MOTS-CLÉS : production des voyelles L2; allemand langue étrangère; français langue maternelle; qualité vocalique.

KEYWORDS: L2 vowel production, German L2, French L1, vowel quality. 


\section{Introduction}

La prononciation en langue étrangère peut différer de la prononciation d'un locuteur natif de la même langue. Nous nous intéressons ici à la prononciation de quelques voyelles longues et brèves allemandes par des germanophones natifs et des apprenants francophones de l'allemand.

A notre connaissance, une étude comparative de la qualité vocalique chez les apprenants francophones de l'allemand et des germanophones natifs n'a pas encore été menée. Cependant, les résultats d'un test de perception réalisé auprès de germanophones natifs amenés à identifier des mots produits par des apprenants francophones de l'allemand ont été publiés (Zimmerer \& Trouvain, 2015). Ce test de perception révèle que les germanophones natifs sont en effet capables de correctement identifier les voyelles produits par les apprenants et cela d'autant plus que les apprenants sont d'un niveau avancé en allemand. En revanche, les voyelles brèves ont été moins souvent identifiées correctement que les voyelles longues. Cette tendance était observée à la fois pour les productions des apprenants débutants et des apprenants avancés.

Dans une étude préalable sur le même corpus qui est étudié dans l'étude présente, nous avons observé que les apprenants francophones de l'allemand n'ont pas de difficultés à produire la différence de durée des voyelles longues et brèves (Wottawa et al., 2018). Nous voulons maintenant analyser la réalisation de la qualité vocalique entre voyelles longues et brèves chez les apprenants francophones de l'allemand et les comparer à des productions chez des germanophones natifs. Pour ce faire, nous avons d'abord comparé les distances euclidiennes des paires vocaliques entre germanophones natifs et des apprenants de l'allemand. Une comparaison similaire a été effectuée entre des locuteurs natifs de l'anglais américain et des apprenants mandarins de l'anglais (Chen, 2006). Ensuite, nous avons comparé les voyelles par paire longue-courte afin de déterminer leurs positions différentes dans l'espace vocalique.

\section{Méthodes}

Dans cette section, nous présentons le corpus (FLACGS) et les analyses acoustiques, incluant le calcul de distances euclidiennes entre voyelles ainsi qu' une comparaison des trois premiers formants.

\subsection{Corpus de parole et matériel acoustique}

Les analyses acoustiques ont été menées sur le French Learners' Audio Corpus of German Speech (FLACGS) Corpus (Wottawa \& Adda-Decker, 2016). Le corpus contient des enregistrements de 40 locuteurs en allemand dont 20 locuteurs germanophones natifs et 20 apprenants de l'allemand ayant le français comme langue maternelle. Le niveau de compétences des apprenants allait de A2 à C2 avec la distribution suivante : A1/A2 : 3 participants, B1/B2 : 9 participants, C1/C2 : 8 participants.

Dans le cadre de cette étude, deux tâches de production ont été analysés : la répétition de mots dans des phrases cadre et la lecture de deux textes allemands (Nordwind und Sonne, Die Buttergeschichte). Dans l'ensemble, le volume de production représente un peu plus que quatre heures de parole. Le corpus a été transcrit manuellement et aligné automatiquement par web-Maus (Kisler et al., 2017). Pour les voyelles, l'alignement automatique a été corrigé manuellement si nécessaire en se basant sur le deuxième formant et ses mouvements. Si la voyelle était suivie d'une consonne voisée ou d'une 
autre voyelle, le choix des frontières de segment était guidé par les mouvements du F2 et de l'intensité relative. La forme du signal acoustique fournissait également quelques indications sur l'emplacement des frontières. Seules les voyelles apparaissant dans des syllabes portant l'accent lexical entraient dans l'analyse des voyelles longues et brèves.

En utilisant Praat (Boersma \& Weenink, 2016), les valeurs formantiques en Hertz des trois premiers formants ont été extraites toutes les $5 \mathrm{~ms}$ à partir des enregistrements audio. Ensuite, elles étaient moyennées pour l'ensemble de la voyelle ainsi que pour le début, le centre et la fin de la voyelle. Pour les analyses décrites par la suite, nous avons utilisé uniquement les valeurs du centre des voyelles transformées en Bark.

\subsection{Analyses acoustiques}

\subsubsection{Distances euclidiennes}

Nous avons calculé les distances euclidiennes entre les voyelles suivantes : [i: $\mathrm{I}],[\mathrm{y}:, \mathrm{y}],[\mathrm{a} ; \mathrm{a}, \mathrm{a}]$ et [O:, o] pour chaque participant. Dans un premier temps, ces distances incluaient uniquement les distances correspondant à F1 et F2 (Traunmüller, 1981). Dans un deuxième temps, nous avons ajouté également la distance euclidienne pour F3.

D'abord, nous avons comparé les distances euclidiennes calculées avec les deux premiers formants à celle calculées avec les trois premiers formants. Ensuite, nous avons établi les relations entre les distances euclidiennes et le groupe de locuteurs (germanophones natifs, apprenants de l'allemand) et les tâches de production (répétition, lecture). Pour ce faire, nous avons eu recours modèle mixte à pente aléatoire (random slope model) (Winter, 2013) en utilisant la library lme 4 (Bates et al., 2015) intégré en R (R Development Core Team, 2008).

\subsubsection{Comparaison des valeurs formantiques}

La distance euclidienne entre les voyelles longues et brèves permet de quantifier les différences de production de la qualité vocalique entre germanophones natifs et apprenants de l'allemand. Cependant, nous avons également effectué des régressions logistiques multiples des trois premiers formants pour chacune des paires vocaliques cf. [i: I], [y:, y], [a: a], [o: , o] afin de mieux déterminer la position des voyelles dans l'espace vocalique. De cette manière, la localisation des deux membres d'une paire vocalique peut être comparée entre les productions des natifs et celle des apprenants.

\section{Résultats}

Dans cette section, nous présentons les résultats de deux analyses. Dans un premier temps, les distances euclidiennes sont présentées et analysées en utilisant un modèle mixte à pente aléatoire. Ensuite, nous comparons les valeurs formantiques des voyelles entre les production des natifs et les apprenants par le moyen de la régression logistique multiple. 


\subsection{Distances euclidiennes}

Tout d'abord, nous avons comparé les distances euclidiennes calculées uniquement avec F1 et F2 à celles calculées avec F1, F2 et F3 en utilisant un test de Student apparié pour chacun des groupes, c'est-à-dire les germanophones natifs et les apprenants de l'allemand et pour chacune des tâches, c'est-à-dire la répétition et la lecture. Les résultats de ces comparaisons ont montré qu'en général, l'ajout de F3 dans le calcul augmente significativement la distance entre les voyelles longues et brèves. Seul pour le contraste [o:, o] chez les germanophones natifs en répétition et les apprenants de l'allemand en lecture, le calcul comportant les valeurs de F3 ne change pas significativement la distance euclidienne. En d'autres termes, ces résultats montrent que dans nos données le F3 joue un rôle important dans la production des voyelles longues et brèves à la fois chez les germanophones natifs et les apprenants de l'allemand. C'est pourquoi les analyses suivantes ont été effectuées avec les distances euclidiennes calculées à partir des trois premiers formants.

Nous avons étudié les relations entre les distances euclidiennes, les groupes de locuteurs (germanophones natifs, apprenants de l'allemand) et les tâches de production (répétition, lecture) à l'aide d'un modèle mixte à pente aléatoire (random slope model). L'importance statistique pour chaque facteur fixe a été calculée par un test du rapport des vraisemblances selon une approche allant d'un modèle simple à un modèle complexe. Les facteurs fixes étaient testés dans l'ordre : Paire vocalique ([i:, I], [y:, y], [a:, a], [o:, o]), Genre du locuteur, Groupe (germanophones natifs, apprenants de l'allemand) et Tâche (répétition, lecture). Nous avons obtenu des intercepts pour le facteur aléatoire Participant ainsi que les interactions Paire vocalique par Participant et Tâche par Participant.

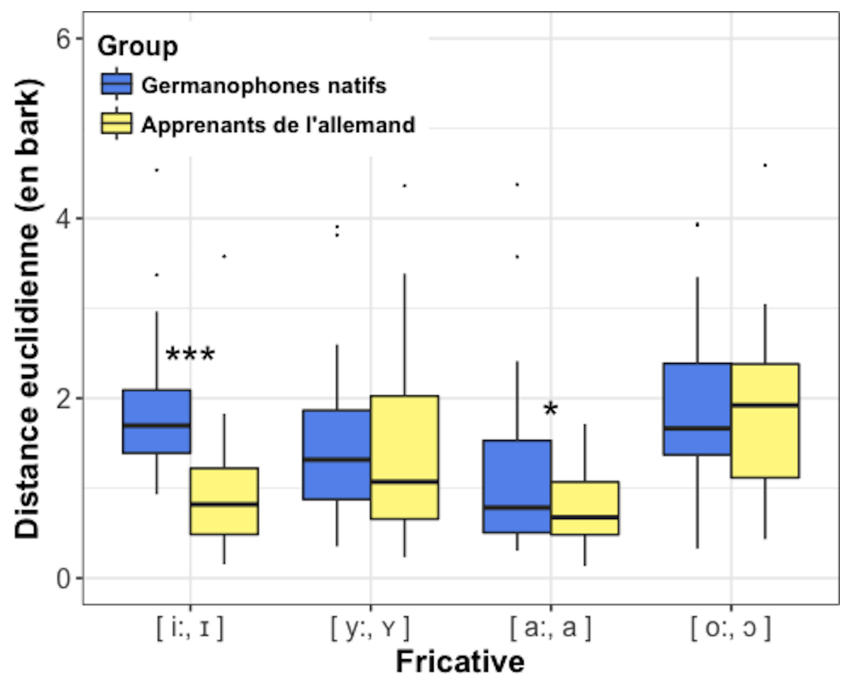

Figure 1: Interaction pour les distances euclidiennes entre Paire vocalique et Groupe ; une différence significative entre groupes est observée pour [i: I] et [a:, a $]\left({ }^{*} p \leq 0.05 ;{ }^{* *} p \leq 0.01 ;{ }^{* * *} p \leq 0.001\right)$

Inclure le facteur Genre n'a pas augmenté la précision du modèle le plus simple qui avait comme seul facteur fixe Paire vocalique $\left(\chi^{2}(1)=0.12, p=.73\right)$. En revanche, le modèle a gagné en précision en incluant le facteur Groupe $\left(\chi^{2}(1)=7.94, p<.01\right)$. Chez les germanophones natifs, les distances euclidiennes sont d'environ 0.5 Bark ( \pm 0.2 Bark) plus grandes par rapport à l'intercept $(0.7$ Bark $( \pm 0.1$ Bark $))$. Ensuite, nous avons testé s'il y a une interaction entre Paire vocalique et Groupe $\left(\chi^{2}(3)=9.83, p<.05\right)$ illustrée par la Figure 1. Par rapport à l'intercept (0.8 Bark $( \pm 0.2$ Bark $\left.)\right)$, les germanophones natifs produisent une distance euclidienne qui est 0.6 Bark ( \pm 0.3 Bark) plus grande pour la paire vocalique [is, I] , et $0.5 \mathrm{Bark}( \pm 0.4 \mathrm{Bark})$ plus petite pour la paire vocalique [y: 
y] et de 0.2 Bark ( \pm 0.4 Bark) plus petite pour la paire vocalique [o:, ৩]. Des comparaisons post-hoc ont révélées que la différence des distances euclidiennes entre Groupe n' est significative que pour les paires vocaliques $[\mathrm{i}, \mathrm{I}](\mathrm{t}(77)=4.3, \mathrm{p}<.001)$ et $[\mathrm{a}, \mathrm{a}](\mathrm{t}(54)=2.5, \mathrm{p}<.05)$. Dans les deux cas, les germanophones natifs ([i, $\mathrm{I}]: M=2.0$ Bark ( \pm 0.9 Bark), [a:, a] : $M=1.2$ Bark ( \pm 0.88 Bark) montrent une distance euclidienne plus importante que les apprenants de l'allemand ([i: $\mathrm{I}]: M=1.8$ Bark ( \pm 1.2 Bark), [a:, a] : $M=0.8$ Bark $( \pm 0.4$ Bark $))$. Finalement, inclure le facteur Tâche ne sert pas à la précision du modèle $\left(\chi^{2}(1)=1.31, p=.25\right)$ c'est pourquoi nous l'avons écarté de l'analyse.

\subsection{Comparaison des valeurs formantiques}

La distance euclidienne entre les voyelles longues et brèves permet de quantifier les différences de production de la qualité vocalique. Cependant, elles ne nous informent pas sur la position des voyelles dans l'espace vocalique. C'est pourquoi nous avons mené une analyse des trois premiers formants par rapport au Groupe (germanophones natifs, apprenants de l'allemand) pour chacune des paires vocaliques, cf. [i:, I], [y: y], [a: a] et [o: o]. Le Groupe était fixé comme la variable dépendante de la régression logistique multiple. Les variables Voyelle, Genre du locuteur, F1, F2, F3 et Tâche (répétition, lecture) étaient les variables explicatives testées dans cet ordre en allant du modèle le plus simple au modèle le plus complexe. Le modèle nul ne comportait que la variable explicative Voyelle.

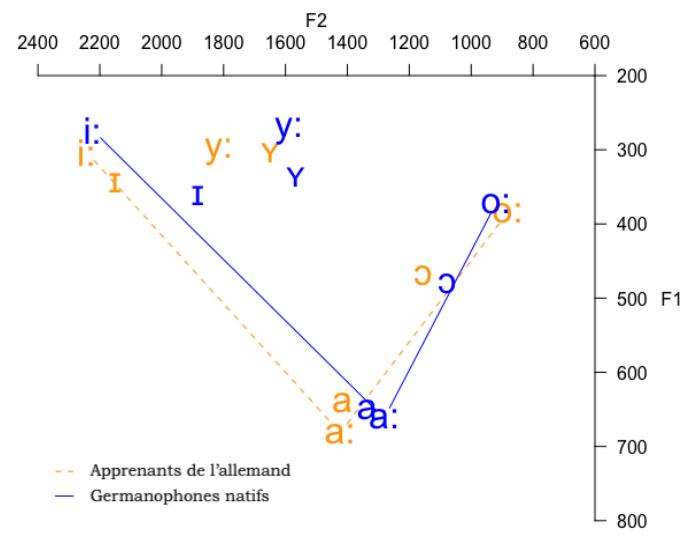

Figure 2: Interaction entre Paire vocalique et F2 par Groupe, espace vocalique F1-F2 en Hz

Les variables Genre de locuteur et Tâche n'ont pas été statistiquement significatives dans les calculs et cela pour aucune paire vocalique. Cependant, les analyses nous informent que pour les paires vocaliques [y: y], [a: a] et [o:, o], la différence entre germanophones natifs et apprenants de l'allemand n'est significative que pour les valeurs du F2 et cela d'autant plus si le F2 entre en interaction avec la variable Voyelle ([y:, y] $:\left(\chi^{2}(1)=7.59, p<.01\right)$; [a: a] $:\left(\chi^{2}(1)=17.12, p<.001\right)$; [o:, o] : $\left.\left(\chi^{2}(1)=5.27, p<.05\right)\right)$. Figure 2 illustre ces interactions. Pour la paire [y:, y], les germanophones natifs ([y] : $M=11.3$ Bark ( \pm 1.1 Bark) ; [y:] : $M=11.0$ Bark ( \pm 3.5 Bark)) ont 1.4 fois plus de chance de produire la voyelle [y: si le F2 baisse d'un bark que les apprenants de l'allemand ([y] : $M=11.4$ Bark ( \pm 2.8 Bark) ; [y:] : $M=12.3$ Bark ( \pm 1.1 Bark)). Pour la paire [ai, a], les germanophones natifs ([a] : $M=10.0$ Bark ( \pm 3.1 Bark) ; [a:] : $M=9.8$ Bark $( \pm 2.3$ Bark)) ont 1.3 fois plus de chance de produire la voyelle [a:] si le F2 baisse d'un bark que les apprenants de l'allemand ([a] : $M=10.6$ Bark ( \pm 1.7 Bark) ; [a:] : $M=10.7$ Bark ( \pm 1.2 Bark)). Pour la paire [o:, o], les germanophones natifs ([o] : $M=8.8$ Bark ( \pm 2.0 Bark) ; [o:] : $M=7.8$ Bark $( \pm 2.1$ Bark $)$ ) ont 1.2 fois plus de chance de produire la voyelle [o: ] si le F2 augmente d'un bark que les apprenants de l'allemand ([o] : $M=9.3$ Bark $( \pm 1.4$ 
Bark) ; [o:] : $M=7.7$ Bark ( \pm 1.4 Bark $))$.

Outre F2, F3 permet de distinguer la paire vocalique [a:' a] entre germanophones natifs et apprenants $\left.\left(\chi^{2}(1)=9.45, p<.05\right)\right)$. Les germanophones natifs $([\mathrm{a}]: M=13.6$ Bark $( \pm 4.1$ Bark $)$; [a: $]$ : $M=14.4$ Bark ( \pm 3.0 Bark)) ont 1,3 fois plus de chance de produire [a:] si F3 augmente d'un bark que les apprenants de l'allemand ([a] : $M=14.6$ Bark ( \pm 2.0 Bark) ; [a: : $M=14.9$ Bark $( \pm 1.0$ Bark)). Les Groupes (germanophones natifs, apprenants de l'allemand) produisent [i: et [I] avec des valeurs différentes pour chacun des trois premiers formants $\left(\mathrm{F} 1: \chi^{2}(1)=28.42, p<.001\right)$; $\left.\left.\mathrm{F} 2: \chi^{2}(3)=75.47, p<.001\right) ; \mathrm{F} 3: \chi^{2}(7)=54.04, p<.001\right)$. Les moyennes pour [i: $]$ et $[\mathrm{I}]$ sont résumés dans Table 1. Le tableau et les résultats des analyses indiquent que par rapport aux apprenants de l'allemand, les germanophones ont 2.1 fois plus de chances de produire [i:] si le F1 baisse d'un bark, 1.3 fois plus de chances de produire [i:] si le F2 augmente d'un bark et 4.7 fois plus de chances de produire [i:] si le F3 baisse d'un bark.

\begin{tabular}{|c|c||c|c||c|c||c|c|}
\hline \multirow{2}{*}{ Groupe } & \multirow{2}{*}{ Voyelle } & \multicolumn{2}{|c||}{ F1 (bark) } & \multicolumn{2}{c||}{ F2 (bark) } & \multicolumn{2}{c|}{ F3 (bark) } \\
\cline { 3 - 8 } & & $M$ & \pm & $M$ & \pm & $M$ & \pm \\
\hline germanophones & {$[\mathrm{I}]$} & 3.6 & 0.9 & 12.4 & 2.2 & 15.0 & 2.3 \\
natifs & {$[\mathrm{i}:]$} & 2.7 & 0.9 & 13.3 & 3.2 & 15.2 & 3.6 \\
\hline apprenants & {$[\mathrm{I}]$} & 3.4 & 0.8 & 13.3 & 1.8 & 15.5 & 1.7 \\
de l'allemand & {$[\mathrm{i}:]$} & 3.0 & 0.7 & 13.7 & 1.4 & 16.0 & 1.3 \\
\hline
\end{tabular}

Table 1: Valeurs moyennes pour F1, F2 et F3 par Groupe en bark

\section{Discussion}

Tout d'abord, nous avons calculé les distances euclidienne pour les paires vocaliques [i: I], [y:, y], [ai, a] et [o: o]. Dans un premier temps, nous avons comparé les distances euclidiennes incluant F1 et F2 à des distances euclidiennes comportant F1, F2 et F3. Nos résultats ont montré que les distances sont plus grandes lorsque le F3 est inclus dans le calcul. Ce résultat peut être expliqué par le fait qu'en allemand les voyelles longues sont soit plus arrondies soit plus étirées que les voyelles courtes. En d'autres termes quant au F3, les voyelles longues se trouvent plus aux extrémités de l'espace vocalique que les voyelles brèves qui sont plus centrales.

Dans un deuxième temps, nous avons étudié les effets des Groupes (germanophones natifs, apprenants de l'allemand), des Tâches de production (répétition, lecture) sur les distances euclidiennes calculées pour chaque participant pour les paires vocaliques [i:, I], [y:, y], [a:, a] et [o:, o]. En général, les germanophones natifs montrent des distances euclidiennes plus grandes que les francophones. Une analyse par paire vocalique a relevé que les distances euclidiennes des germanophones natifs ne sont significativement plus importantes que pour les paires vocaliques [ii, $\mathrm{I}]$ et [a: a]. Ces résultats sont un premier indice des différences qui existent entre les productions vocaliques des germanophones natifs et des apprenants de l'allemand. Les voyelles [i: et [I] ainsi que [a:] et [a] semblent moins bien séparées chez les apprenants de l'allemand que chez les germanophones natifs. Le genre des locuteurs et la tâche n'avaient pas d'influence sur les distances euclidiennes. En d'autres termes, les locuteurs féminins et masculins appartenant à un des deux groupes produisent des distances similaires. Les distances ne sont pas affectées par la tâche ce qui suggère qu'aucune des deux tâches est plus facile ou plus complexe pour la production des voyelles en langue étrangère. 
Les régressions logistiques ont été menées dans l'objectif de mieux caractériser les différences de la qualité vocalique qui existent entre les productions des locuteurs natifs et des apprenants. Pour ces analyses aussi, le genre des locuteurs et la tâche de production n'ont pas influencé la production des voyelles entre les deux groupes. Que le genre ne joue pas de rôle significatif peut être expliqué par le fait que le nombre d'hommes et de femmes enregistrés était équivalent dans les deux groupes.

Les analyses de régression logistique ont relevé que les productions des deux groupes se distinguent surtout par les valeurs de F2. Les tendances suivantes se profilent dans nos résultats : pour les paires vocaliques [ir, I] et [oi, o], le F2 des deux groupes est comparable pour les voyelles longues. Cependant, pour les paires vocaliques [y:, y] et [a: a] le F2 des deux groupes est comparable pour les voyelles brèves. Les germanophones natifs produisent la voyelle [I] bien plus centrale que les apprenants dont le F2 de leurs productions de [I] est presque identique à leurs productions de [i:], donc antérieur. Dans l'espace vocalique, les productions du [y:], [a:], [a] et [o] des apprenants occupent également une position plus antérieure que celle des germanophones natifs. De plus, pour les paires centrales $[\mathrm{y}: \mathrm{y}]$ et $[\mathrm{a}: \mathrm{a}]$, les apprenants francophones ont tendance à produire les voyelles longues plus antérieures que leurs contre-parties brèves. Chez les germanophones natifs, les voyelles centrales tendues sont légèrement plus postérieures que leurs contre-parties relâchées.

Seulement deux paires vocaliques ont montré des différences significatives entre les deux groupes quant aux valeurs du F3 : [ii, I] et [a: a]. Les valeurs de F3 sont plus élevées pour [i:] que pour [I] chez les apprenants francophones de l'allemand. Cependant, les valeurs de F3 des deux voyelles sont presque identiques chez les germanophones natifs. Il est possible que les apprenants francophones de l'allemand soient conscient de la différence entre [ii] et [I] et essaient de la marquer en produisant un [I] qui est moins étiré que [i:]. Leurs productions de [a: et [a] montrent des valeurs de F3 quasiment identiques alors que chez les germanophones natifs, le F3 est plus élevé pour [a:] que pour [a]. Les différences du F3 entre les deux groupes pour la paire vocalique [a:, a] pourrait expliquer les différences des distances euclidiennes présentées plus haut.

Seule la paire vocalique [ir, I] permet de distinguer les locuteurs des deux groupes par le F1. En moyenne, chez les germanophones natifs le F1 diffère d'un bark entre [i:] et [I]. Chez les apprenants, la différence n'est que de 0.4 Bark. Dans l'espace vocalique des apprenants de l'allemand, la place restreinte occupée par [ii] et [I] permet une place relativement antérieure pour la voyelle [y:]. Chez les germanophones natifs, au contraire, la distance entre [ii] et [I] est grande engageant surtout les deux premiers formants. En revanche,la distance entre [y: et [Y] est plus faible surtout concernant les valeurs du F2.

\section{Conclusion}

Nos analyses nous ont permis de caractériser les différences de production des voyelles longues et brèves de l'allemand ([i: , I], [y: y], [a: a], [o: o]) entre les locuteurs germanophones natifs et les apprenants de l'allemand enregistrés dans le corpus FLACGS. Cette comparaison systématique des distances euclidiennes et des analyses formantiques en fonction des deux groupes de locuteurs a indiqué que la paire vocalique [i: I] présente les différences acoustiques les plus importantes entre les deux groupes de locuteurs. Les différences concernent à la fois la distance euclidienne qui est plus petite chez les apprenants de l'allemand que chez les germanophones natifs et les valeurs des trois premiers formants. Cependant, dans l'ensemble des paires vocaliques choisies, la paire [i: , I] est quelque peu atypique. Pour les autres paires vocaliques, les deux groupes de locuteurs se différencient 
surtout par les valeurs du F2. Les apprenants francophones ont tendance à produire des voyelles avec des valeurs de F2 plus élevées que les germanophones natifs.

Les résultats de nos analyses acoustiques ne permettent malheureusement pas d'expliquer les résultats du test de perception réalisé auprès de germanophones natifs (Zimmerer \& Trouvain, 2015). Ce test indiquait que les voyelles [Y] et [o] étaient les moins bien identifiées alors que dans notre corpus, les voyelles natives et non-natives [Y] et [o] sont relativement proches et surtout bien distinctes de [y: et [o:]. Il serait intéressant de mener les mêmes analyses acoustiques présentées en haut sur les stimuli utilisés lors du test de perception afin de comparer ces productions aux productions analysées ici.

\section{Remerciements}

Ce travail a été soutenu par le programme Investissements d'Avenir - Labex EFL (ANR-10-LABX-0083).

\section{References}

Bates D., Mächler M., Bolker B. \& Walker S. (2015). Fitting linear mixed-effects models using lme4. Journal of Statistical Software, 67(1), 1-48.

Boersma P. \& WeEnINK D. (2016). Praat: doing phonetics by computer [Computer program]. Version 6.0.19, retrieved 13 June 2016.

CHEN Y. (2006). Production of tense-lax contrast by Mandarin speakers of English. Folia phoniatrica et logopaedica, 58(4), 240-249.

KISLER T., REICHEL U. \& SCHIEL F. (2017). Multilingual processing of speech via web services. Computer Speech \& Language, 45, 326 - 347.

R Development Core Team (2008). R: A Language and Environment for Statistical Computing. R Foundation for Statistical Computing, Vienna, Austria. ISBN 3-900051-07-0.

Traunmüller H. (1981). Perceptual dimension of openness in vowels. The Journal of the Acoustical Society of America, 69(5), 1465-1475.

WINTER B. (2013). A very basic tutorial for performing linear mixed effects analyses. arXiv preprint arXiv:1308.5499.

Wottawa J. \& Adda-Decker M. (2016). French Learners Audio Corpus of German Speech (FLACGS). In Proceedings of the 10th International Conference on Language Resources and Evaluation (LREC'16), Portorož (Slovenia).

Wottawa J., AdDA-Decker M. \& Isel F. (2018). The impact of production complexity in German L2 by French native speakers: Focus on /h/ and vowel duration contrast. In E. BABATSOULI \& D. Ingram, Eds., Phonology in Protolanguage and Interlanguage. Equinox.

Zimmerer F. \& Trouvain J. (2015). Perception of French speakers' German vowels. Interspeech 2015 . 